\title{
ANÁLISIS DE LA RED DE COMUNICACIÓN DEL SUR DE LA CIUDAD DE MURCIA MEDIANTE SIG: LA INTEGRACIÓN DE LA ALTA VELOCIDAD FERROVIARIA Y SU INFLUENCIA
}

\author{
Iván Gil Alonso ${ }^{1}$ \\ Universidad de Murcia
}

\begin{abstract}
RESUMEN
En este artículo se evalúa la vulnerabilidad de la red de carreteras del sur del municipio de Murcia ante la inserción del trazado ferroviario de la línea de Alta Velocidad, determinando las variaciones de tiempo en los desplazamientos con origen en los barrios y pedanías situados al sur de la vía ferroviaria hacia los lugares de mayor interés de la ciudad de Murcia. En la metodología se emplean Sistemas de Información Geográfica (SIG) para generar modelos de la red de transporte del municipio de Murcia referidos al escenario previo a la integración de la alta velocidad, escenario de inserción en superficie y escenario de inserción soterrada, para dos tipos de movilidad: a pie y mediante vehículo motorizado. Los costes de tiempo de desplazamiento obtenidos de cada modelo se analizan y comparan estadísticamente obteniendo las variaciones. Los resultados revelan que la inserción en superficie implicaría un incremento de coste en los desplazamientos, mientras que la inserción soterrada reduciría los tiempos de desplazamiento y mejoraría la accesibilidad.

Palabras clave: Red de carreteras; alta velocidad ferroviaria; sistemas de información geográfica (SIG); vulnerabilidad; territorio.

\section{ANALYSIS OF THE COMMUNICATION NETWORK OF THE SOUTH OF MURCIA CITY THROUGH SIG: THE INTEGRATION OF THE HIGH RAIL SPEED AND ITS INFLUENCE}

\section{ABSTRACT}

This article assesses the vulnerability of the road network in the south of the municipality of Murcia to the insertion of the railway line of the High-Speed line, determining the time variations in movements originating in the neighborhoods and districts located south of the railway to the places of greatest interest in the city of Murcia. In the methodology, Geographic Information Systems (GIS) are used to generate models of the transport network of the municipality of Murcia referring to the scenario prior to the integration of high speed, scenario of surface insertion and scenario of underground insertion, for two types mobility: on foot and by motorized vehicle. The travel time costs obtained from each model are statistically analyzed and compared obtaining the variations. The results reveal that surface insertion would imply an increase in travel costs, while underground insertion would reduce travel times and improve accessibility.

Key words: Road network; high-speed rail; geographic information systems (GIS); vulnerability; territory.

\footnotetext{
1 ivan.zikitx@gmail.com
}

Fecha de recepción: 11 de octubre de 2020. Fecha de aceptación: 11 de enero de 2021 


\section{INTRODUCCIÓN}

El ferrocarril ha brindado desde su aparición en las ciudades españolas, a partir de mitad del siglo XIX, la posibilidad de un servicio de transporte de escasa agresividad con el medio ambiente y ocupación relativa al suelo, alta eficacia energética, destinado hacia las mayorías e idóneo para solventar con eficacia las demandas masivas o intensas de desplazamiento, siendo además complementario con otros medios de transporte (Santos, 2007). Pese a estos aspectos, la existencia o mejora de este servicio ha resultado problemática en numerosas ciudades europeas por motivar cambios en la movilidad urbana y en el funcionamiento del territorio (Álvarez, 2016).

El crecimiento de las ciudades tiene como consecuencia el salto del trazado ferroviario y la expansión de la ciudad al otro lado de la vía caracterizándose en muchos casos por la falta de inserción del ferrocarril en el tejido urbano provocando dificultades en la articulación de la red de transporte por carretera y fractura de la trama urbana, (Pérez, 1994).

Las tradicionales soluciones dadas a esta problemática desde el planeamiento urbanístico local ha sido trazar un nuevo recorrido para las vías por el exterior del núcleo de población, o el soterramiento de la estación y de toda la travesía urbana ferroviaria (Pérez, 1994).

La ciudad de Murcia, al igual que muchas otras ciudades españolas, se ha visto cohibida en su expansión en primer lugar por el río y posteriormente por la vía del ferrocarril, extendiéndose más allá de estos elementos ante la necesidad de nuevas viviendas (Álvarez y Hernández, 2012). Para el caso de los vecinos situados más allá de las vías, éstas han incidido históricamente dificultando la articulación de los barrios periféricos con el resto de la ciudad como circunstancia del efecto barrera que las vías ejercen (Bellet, et al., 2010).

El transporte por carretera mediante el empleo del automóvil privado se ha convertido en el modo hegemónico, tanto para el desplazamiento de personas como de mercancías. Por ello, las interrupciones del tráfico en determinados tramos de la red, debido a la influencia del trazado ferroviario, conlleva consecuencias económicas y sociales desfavorables (Bono y Gutiérrez, 2011).

La llegada del tren de alta velocidad a la ciudad de Murcia constituye una oportunidad para revertir la relación de la infraestructura ferroviaria con el espacio urbano. La integración del ferrocarril en el sistema urbano, finalmente, se llevará a cabo mediante el soterramiento de las vías a su paso por el núcleo urbano, aprovechando de esta manera la estructura ferroviaria y el trazado existente y recuperando los barrios colindantes evitando así su marginación y degradación (Bellet y Gutiérrez, 2011). Sin embargo, inicialmente se planteó la inserción de la alta velocidad ferroviaria (AVF) en superficie.

Por tanto, la implantación de la AVF en la ciudad de Murcia ofrecía dos posibles escenarios bien diferenciados, el de la integración de la AVF soterrada o en superficie.

En el presente artículo se analiza la vulnerabilidad del territorio, a partir de la red de transporte de la ciudad de Murcia, ante la alteración que supondría la inserción de la AVF en superficie. Se evalúan las consecuencias derivadas de la supresión o bloqueo temporal de varios tramos, determinando el previsible incremento en los tiempos de viaje deducidos de la necesidad de recurrir a rutas alternativas a la óptima.

El estudio de redes se realiza mediante representaciones teóricas y metodológicas basadas en la formulación matemática de la Teoría de Grafos (Newman, 2010). El análisis de las propiedades de conectividad de redes reales de gran tamaño inició el camino hacia el estudio de redes complejas, entre las que se encuentran las redes de transporte. El empleo de Teoría de Grafos en sistemas de transporte urbano apareció entre los años 50 y 70 con la finalidad de conocer los impactos económicos regionales en los sistemas de autopistas interestatales en los Estados Unidos (Derrible y Kennedy, 2011).

Los análisis de vulnerabilidad están enfocados a analizar cómo se vería afectada la red y el territorio ante accidentes de tráfico, desastres naturales, cierres programados de tramos, o cualquier otro evento que implique, en términos de redes, la eliminación de uno o varios arcos de la red, y que pudiera afectar la conectividad de ésta (Vélez y Heredia, 2014).

No se ha dado consenso en la definición del concepto de vulnerabilidad, variando en función del área del conocimiento y del contexto. Nagurney y Qiang (2011) contemplan el análisis de 
vulnerabilidad de redes complejas como el método de cuantificar y evaluar el impacto de la supresión de un elemento de la red. Similar es la definición aportada por Gol'dshtein, Koganov, y Surdutovich (2004), para ellos la vulnerabilidad en redes consiste en la reducción del desempeño de la red dada la supresión de un vértice junto con los arcos que lo conectan con otros vértices. Generalmente la vulnerabilidad de la red se evalúa tras la eliminación de uno o varios elementos (nodos y/o arcos).

La morfología y la densidad de la red son factores considerables, dado que una red densa, descentralizada, mallada, con gran número de arcos, facilita los desplazamientos y permite multitud de rutas alternativas, mientras que una red poco densa refuerza el contratiempo (Cova, 1999).

El objetivo de este artículo es presentar y aplicar una metodología para el análisis de redes de carreteras mediante las funcionalidades de los Sistemas de Información Geográfica (SIG). Consiste en estudiar los efectos derivados de los cierres o bloqueos temporales en ciertos tramos. Debido al efecto red, estos efectos se extienden sobre un espacio mucho mayor al referido al arco directamente afectado. Los efectos se miden en términos de aumento de tiempos de viaje, lo que supone pérdidas de accesibilidad. Se analiza tanto la intensidad de los efectos como su grado de polarización en el territorio, realizándose una comparación entre los diferentes escenarios obteniéndose las variaciones que permiten extraer conclusiones acerca de los efectos sobre el territorio derivados de la inserción de la AVF, tanto para los desplazamientos en vehículo como a pie.

El análisis de redes de transporte es un tema de investigación vigente en los estudios actuales, Rodríguez y Gutiérrez (2012) aseguran que el grueso de trabajos sobre esta temática se ha ido produciendo en las dos últimas décadas y por ello, la bibliografía sobre este ámbito no llega a ser todavía muy abundante. Los antecedentes se inician con el precursor trabajo de Garrison (1960), en el que se analizan los incrementos de distancias topológicas a causa de la interrupción del tráfico en determinados tramos de la red de carreteras interestatales de los Estados Unidos, siguieron los estudios de Ratliff et al. (1975) y Ball et al. (1989), quienes establecieron algoritmos para determinar los arcos más "vitales" (críticos) de una red. Es a partir de los terremotos de Los Ángeles de 1994 y Kobe de 1995 y los ataques terroristas de Nueva York, Madrid y Londres, cuando las investigaciones sobre análisis de redes de transporte logran un mayor desarrollo, impulsados por la mayor disponibilidad y eficacia de herramientas informáticas, destacando los Sistemas de Información Geográfica.

Los estudios sobre análisis de la vulnerabilidad de redes de transporte pueden ser clasificados en diferentes categorías, en función de su enfoque (Murray et al., 2008): evaluación de escenarios específicos, identificación de escenarios más negativos y evaluación de escenarios de estrategias de ataques coordinados.

La primera categoría engloba trabajos referidos al análisis de uno o varios escenarios reales o hipotéticos para evaluar los efectos de la interrupción de uno o varios elementos de la red. Se contempla un número reducido de escenarios posibles, permitiendo realizar un análisis detallado de cada uno de ellos. Como ejemplo se puede citar el trabajo de Berdica y Mattsson (2007) en el que se evalúan los impactos del cierre de distintos puentes en Estocolmo. Por su parte, Taylor et al. (2006) analizan los efectos de la supresión de distritos tramos de la red de carreteras australiana en términos de aumento del coste generalizado de transporte y pérdida de accesibilidad. Los trabajos de Knoop et al. (2008) acerca de la red viaria de Rotterdam, al igual que Erath et al. (2008) sobre la red de carreteras de Suiza también se centran en la simulación de impactos a consecuencia del cierre de tramos específicos. Rodríguez (2001) realiza un análisis mediante SIG del impacto de accesibilidad de la línea de alta velocidad Madrid-Barcelona-frontera francesa. Cabe señalar, que también en américa latina se han elaborado trabajos en relación a esta línea, destacando el de Gradilla-Hernández et al. (2011).

El segundo grupo de investigaciones se ocupa de identificar los escenarios más negativos, como ejemplo cabría citar el trabajo de Jenelius y Mattsson (2005) en el cual analizan los efectos de la pérdida de los arcos de una red, con el objetivo de obtener un rango de variabilidad de dichos efectos. En el caso de Murray et al. (2008), se persigue determinar los escenarios más graves dada las combinaciones de pérdidas de varios arcos en la red de carreteras de Ohio. 
Por último, otros trabajos se refieren a la simulación de escenarios de ataques a las redes. En los que se plantea una serie de pérdidas de elementos de la red con la finalidad de conocer la vulnerabilidad de la red ante la pérdida de tales elementos, cabe citar como ejemplo de este tipo de investigación el trabajo de Albert et al. (2000).

El presente trabajo se corresponde con el primer tipo de investigación, dedicado a analizar la vulnerabilidad de las redes de transporte en términos de aumento de costes, generando escenarios hipotéticos y reales de interrupción del tráfico en varios tramos, para analizar comparativamente los impactos derivados, empleando para ello herramientas SIG.

\section{METODOLOGÍA}

\section{1. ÁREA DE ESTUDIO}

El área de estudio queda delimitada por el tramo de actuación de soterramiento de las vías ferroviarias a su paso por el municipio de Murcia, basado en el proyecto de Murcia Alta Velocidad S. A., la sociedad creada mediante convenio entre el Ministerio de Fomento, el Gobierno de la Región de Murcia, el Ayuntamiento de Murcia y el Administrador de Infraestructuras Ferroviarias para la remodelación de la red arterial ferroviaria de la ciudad de Murcia.

El área de estudio comprende un espacio contenido en el municipio de Murcia. Engloba los barrios de Santiago el Mayor, Barriomar, San Pío X y se extiende hacia el sur abarcando la huerta y sus entidades urbanas hasta la serranía de Carrascoy y El Valle. Correspondiéndose con una superficie de 103,6 km2 que representa un 11,7\% del municipio cubriendo una población de 113.065 habitantes según el Censo de Población y Viviendas de 2011 (Instituto Nacional de Estadística). Por tanto, el área de estudio abarca la red de transporte del municipio localizada al sur de la línea ferroviaria.

FIGURA 1

Representación del área de estudio

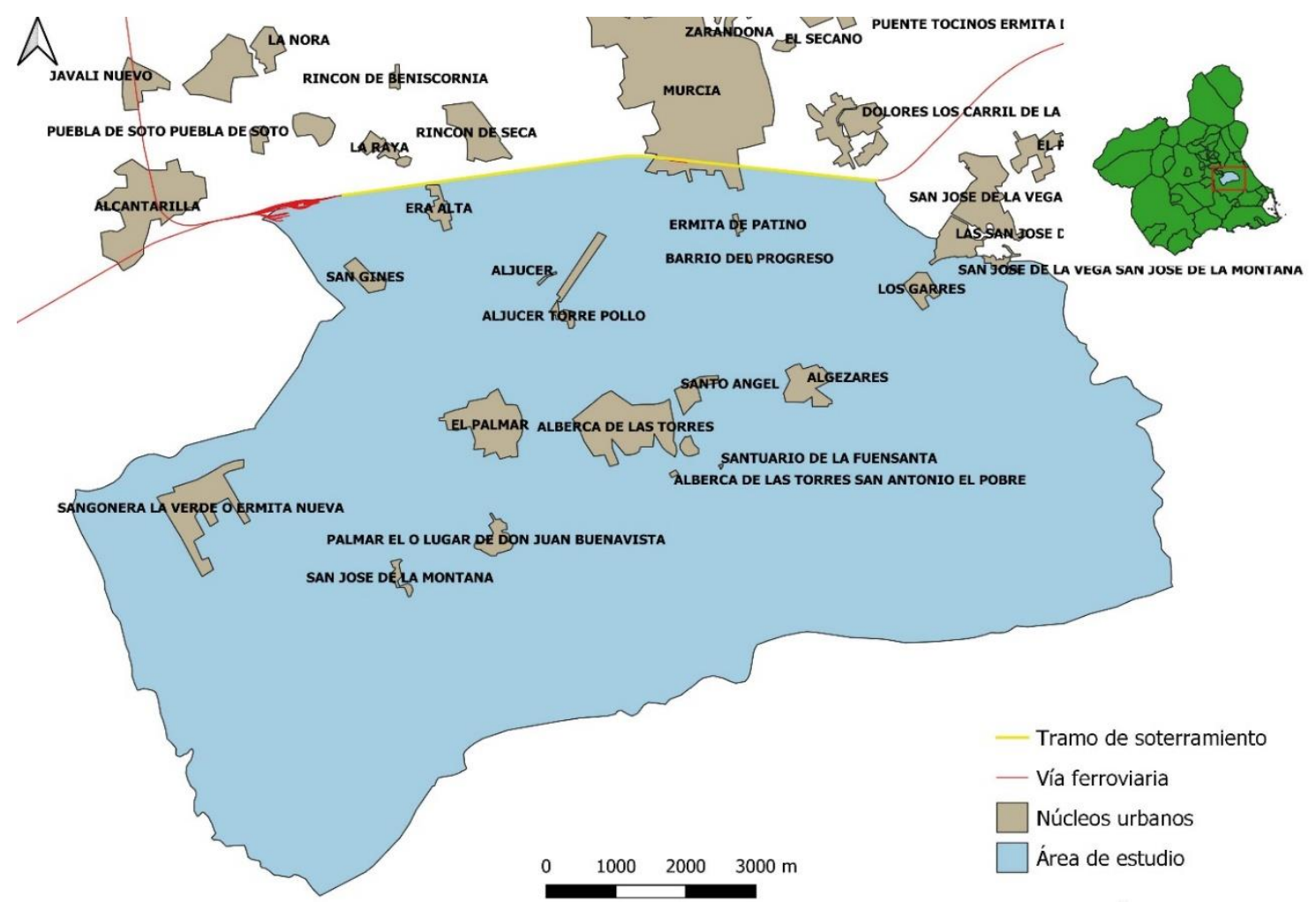

Fuente: Elaboración propia 
La estructura urbanística de esta área de estudio, de gran complejidad, se fundamenta principalmente en la ciudad de Murcia, la cual actúa como referente de la actividad y a partir de la que han ido aflorando diversos núcleos con propósitos fundamentalmente agrícolas (Ros, et al., 2010). En muchos municipios de la Región de Murcia y de todo el país, a estos núcleos o entidades urbanas infra municipales se les denomina habitualmente pedanías (Bel y Gómez, 1998), por tanto, en este trabajo se empleará este mismo término para designarlas. Reconociendo en nuestra área de estudio las de La Alberca, Algezares, Aljucer, Barrio del Progreso, El Palmar, Era Alta, Los Garres, Monte Liso, San Ginés, San José de la Montaña, Sangonera la Verde, Santo Ángel, Patiño y San José de la Vega.

En el suelo dedicado tradicionalmente a uso agrario, comprendido en el área de estudio, se viene observando desde mediados del siglo XX un progresivo descenso de las funciones agrícolas, en favor de los usos residenciales y terciarios, llevándose a cabo un proceso de rururbanización, por el cual se transforma la vida en los campos próximos a grandes ciudades, dada la incorporación al medio rural tradicional de las prácticas sociales y actividades vinculadas al modo de vida urbano (García, 2012), conllevando un desarrollo constructivo y demográfico.

\section{FIGURA 2}

Comparativa con Ortofoto del sur de la ciudad de Murcia y la huerta en los años 1956 (izquierda) y 2016 (derecha).
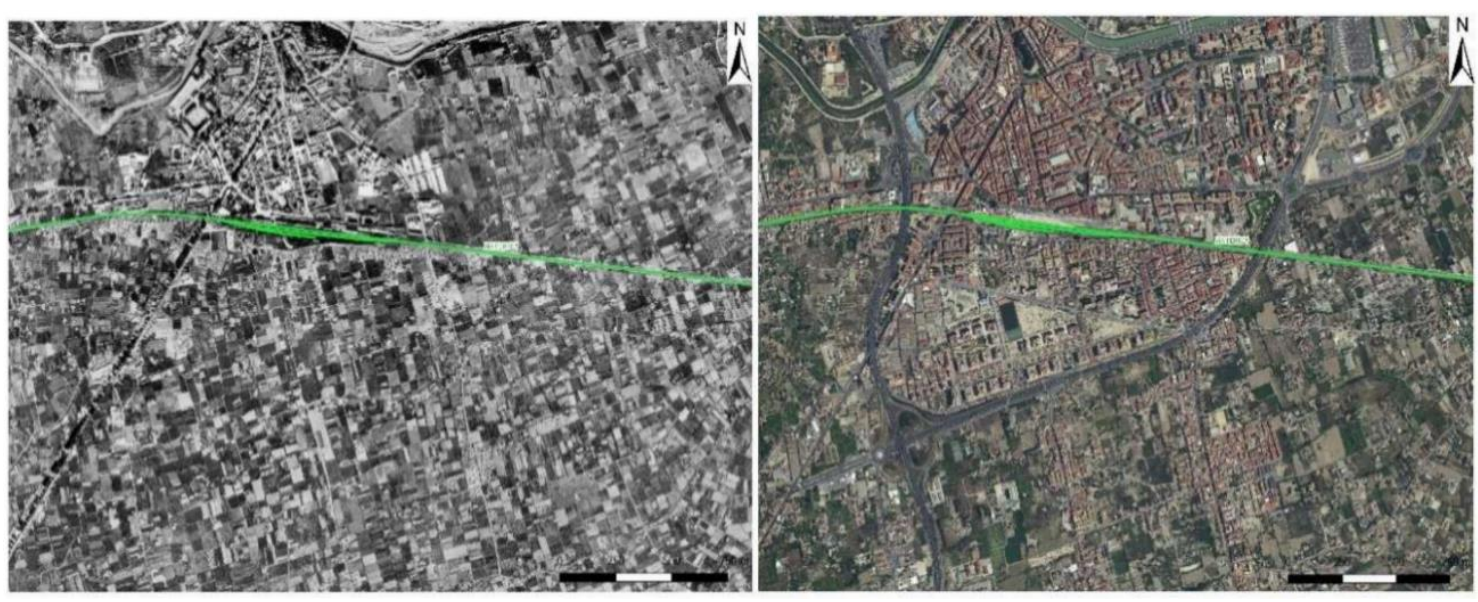

Fuente: Elaboración propia

Como muestra de este proceso y desarrollo, se observa que la suma de la población de las pedanías huertanas de la Alberca, Algezares, Los Garres, El Palmar, Sangonera la Verde y Santo Ángel ha crecido un 14,1\% en el periodo de 2006 a 2016.

TABLA 1

Evolución de la población entre 2006 y 2016

\begin{tabular}{|l|r|r|r|r|r|r|r|}
\cline { 2 - 8 } \multicolumn{1}{c|}{} & La Alberca & Algezares & Los Garres & El Palmar & Sangonera la Verde & Santo Ángel & Total pedanías \\
\hline Población 2006 & 11449 & 4814 & 6033 & 20703 & 8824 & 5295 & 57118 \\
\hline Población 2016 & 12345 & 5481 & 7285 & 22996 & 11227 & 5831 & 65165 \\
\hline Evolución & 896 & 667 & 1252 & 2293 & 2403 & 536 & 8047 \\
\hline
\end{tabular}

Fuente: Elaboración propia mediante datos del Centro Regional de Estadística de Murcia

La transformación de ambiente agrícola a espacio rururbano, supone la intensificación de la movilidad de la población con los polos de concentración de servicios, localizados en el núcleo principal (García, 2012), la ciudad de Murcia.

El espacio de huerta comprendido en el área de estudio presenta pues una elevada densidad de edificación, así como una parcelación de tipo fragmentada y dispersa, precisando de una densa 
red de vías de comunicación, que conecte los distintitos espacios de huerta y pedanías con la ciudad. Esta articulación se compone de una vasta red de carreteras convencionales que integran la huerta, no obstante, destaca considerablemente la presencia de un gran eje que atraviesa en dirección Norte-Sur el área de estudio, se trata de la autovía A-30 que ejerce de conexión rápida entre las pedanías del suroeste y centro del área de estudio (Sangonera la Verde, El Palmar, La Alberca y Santo Ángel) con la ciudad.

FIGURA 3

Red de transporte en el municipio de Murcia

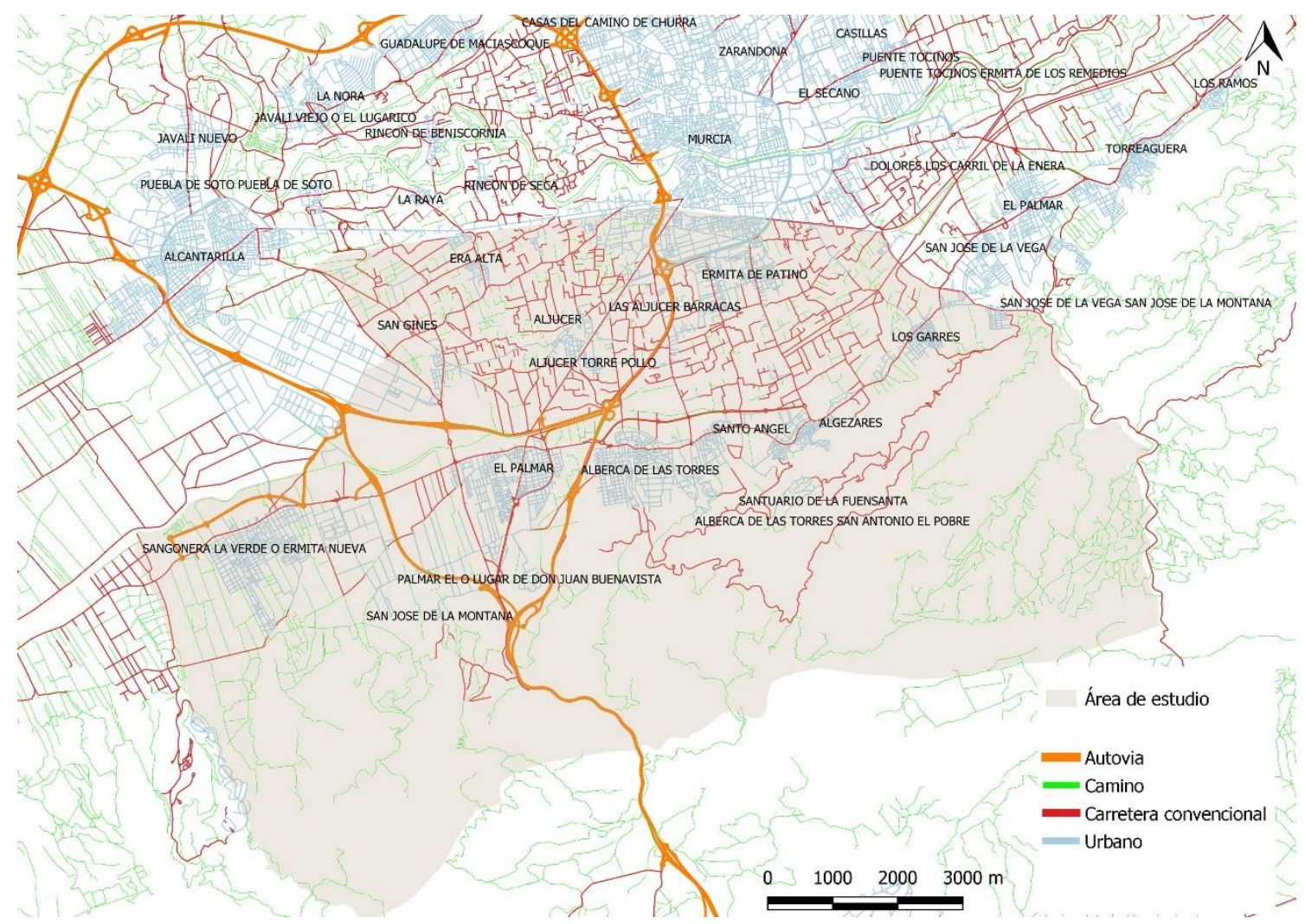

Fuente: Elaboración propia mediante datos obtenidos del Centro de Descargas del Centro Nacional de Información Geográfica

\subsection{ASPECTOS GENERALES}

Para determinar la vulnerabilidad de la red, se elaboran escenarios hipotéticos que representan la red de transporte del municipio de Murcia, en función de dos modalidades: para peatones y para vehículos motorizados. Mediante el empleo de herramientas SIG se desarrollan modelos, empleando Teoría de grafos, que son analizados comparativamente.

En Teoría de grafos un modelo se constituye mediante puntos denominados vértices (o nodos) conectados entre sí mediante líneas denominadas aristas. Por lo que un grafo (G) se puede expresar como $\mathrm{G}=(\mathrm{V}, \mathrm{A})$ donde $\mathrm{V}$ es el conjunto de vértices, y A es el conjunto de aristas (Restrepo y Sánchez, 2004).

\subsection{DATOS}

La red de transporte empleada en los modelos de esta investigación se ha obtenido de la Red de Transporte (RT) por carretera de la Región de Murcia, desde la fuente del Centro de Descargas del Centro Nacional de Información Geográfica (CNIG). Este producto recoge el conjunto de vías de comunicación de la Región de Murcia actualizadas al año 2017. 
El modelo comprende 35.745 aristas, siendo 35.619 el número de aristas que componen la red para la movilidad mediante vehículo de motor. El número difiere en función del tipo de movilidad como consecuencia de las vías de tránsito exclusivo para peatones y viceversa.

La red de transporte del área de estudio se compone de 890 kilómetros de vías en el modelo de movilidad mediante vehículo, y de 880 kilómetros de vías en el modelo de movilidad peatonal.

En el modelo de movilidad mediante vehículo a motor, cada arista reúne información acerca de su longitud, sentido de circulación, jerarquía de la vía, coordenada $\mathrm{Z}$ del viario y coste de tiempo (en segundos) que implica recorrerla. Este coste viene determinado por la longitud del tramo y la velocidad máxima permitida en este.

Sin embargo, en el modelo de movilidad peatonal, no se tiene en cuenta la información sobre sentido de circulación, así como la velocidad se estima en $5 \mathrm{~km} / \mathrm{h}$ para todos los tramos.

Los desplazamientos parten de 22.817 portales localizados en el área de estudio (nodos origen), dirigidos a 38 destinos, seleccionados en base a su cualidad de atraer población e importancia pública. Por último, se establecen 6 nodos barrera, que, en función del escenario representado, ejercerán una determinada influencia sobre el modelo.

\subsection{ESCENARIOS}

Para determinar la vulnerabilidad de la red y el territorio ante la inserción de la AVF, se efectúa un análisis de costes de desplazamiento comparando el escenario anterior a la inserción con cada uno de los hipotéticos escenarios de integración de la alta velocidad. El escenario anterior a la inserción, considerado como escenario de referencia, representa la situación de funcionamiento habitual de la red, previa al proyecto de llegada de la AVF; mientras que los escenarios hipotéticos representan, por un lado, la inserción soterrada de las vías ferroviarias, y por otro, la integración en superficie de las vías.

En el escenario anterior o previo a la integración de la alta velocidad, se da la existencia de una vía ferroviaria en superficie para trenes convencionales, con pasos a nivel que comunican los distritos de un lado y otro de la vía ferroviaria. La característica de este escenario es el coste de tiempo añadido que supone atravesar los pasos a nivel a consecuencia de que la barrera se encuentre bajada por el tránsito ferroviario. Por tanto, en la modelización de este escenario se incluirán los pasos a nivel representados mediante nodos barrera que llevarán asignado un coste agregado (en segundos) correspondiente a los tiempos de espera a causa de la circulación de trenes. Este coste agregado o añadido se calcula a partir del número de trenes que semanalmente llegan y parten de la estación de Murcia del Carmen multiplicado por el tiempo que la circulación a través del paso está cortada a causa del tránsito de un tren (barrera bajada), este valor se divide entre el número de minutos que tiene una semana.

La representación del escenario de inserción en superficie se ha realizado en base al proyecto de la sociedad Murcia Alta Velocidad S.A. Este proyecto supone la llegada de la AVF en superficie de manera provisional para posteriormente llevar a cabo un soterramiento de las vías ferroviarias. Señala que la integración en superficie de la AVF en la ciudad de Murcia supondrá la eliminación de los pasos a nivel, suprimiendo esta conexión para los vehículos motorizados, y la instalación de pasarelas elevadas, por encima de las vías, en estos pasos, que permitirían el tránsito de peatones, aunque con un incremento en el coste de tiempo. Este incremento se calcula similar al incremento que supone atravesar las vías por la pasarela subterránea de calle Floridablanca con carretera del Palmar con respecto al paso a nivel peatonal allí situado. No obstante, la catenaria correspondiente al AVE obligaría a que la altura de la pasarela deba salvar un mayor desnivel que en el caso de este paso subterráneo, por lo tanto, la longitud de esta pasarela elevada sería mayor que la longitud de la pasarela subterránea actual de calle Floridablanca, considerando que pese a que la longitud de esta pasarela constituye un buen ejemplo, cabría esperar un aumento respecto a la longitud de ésta en las pasarelas en altura para salvar las vías y catenarias del AVE. Por tanto, el modelo de movilidad peatonal viene también definido por los pasos a nivel, representados por nodos barrera de coste agregado. No obstante, en el modelo de movilidad mediante vehículo se establecen nodos barrera de restricción, suprimiendo estas conexiones. 
El proyecto de la sociedad Murcia Alta Velocidad S.A. prevé tras implantar la AVF en superficie, llevar a cabo un posterior soterramiento de las vías ferroviarias a su paso por la ciudad de Murcia. El proyecto de Murcia Alta velocidad S.A. supone 7.120 metros de vías soterradas, consistiendo en 7.882 metros de vías ferroviarias implicadas por el tramo de actuación, teniendo en cuenta las rampas.

FIGURA 4

Tramo de soterramiento

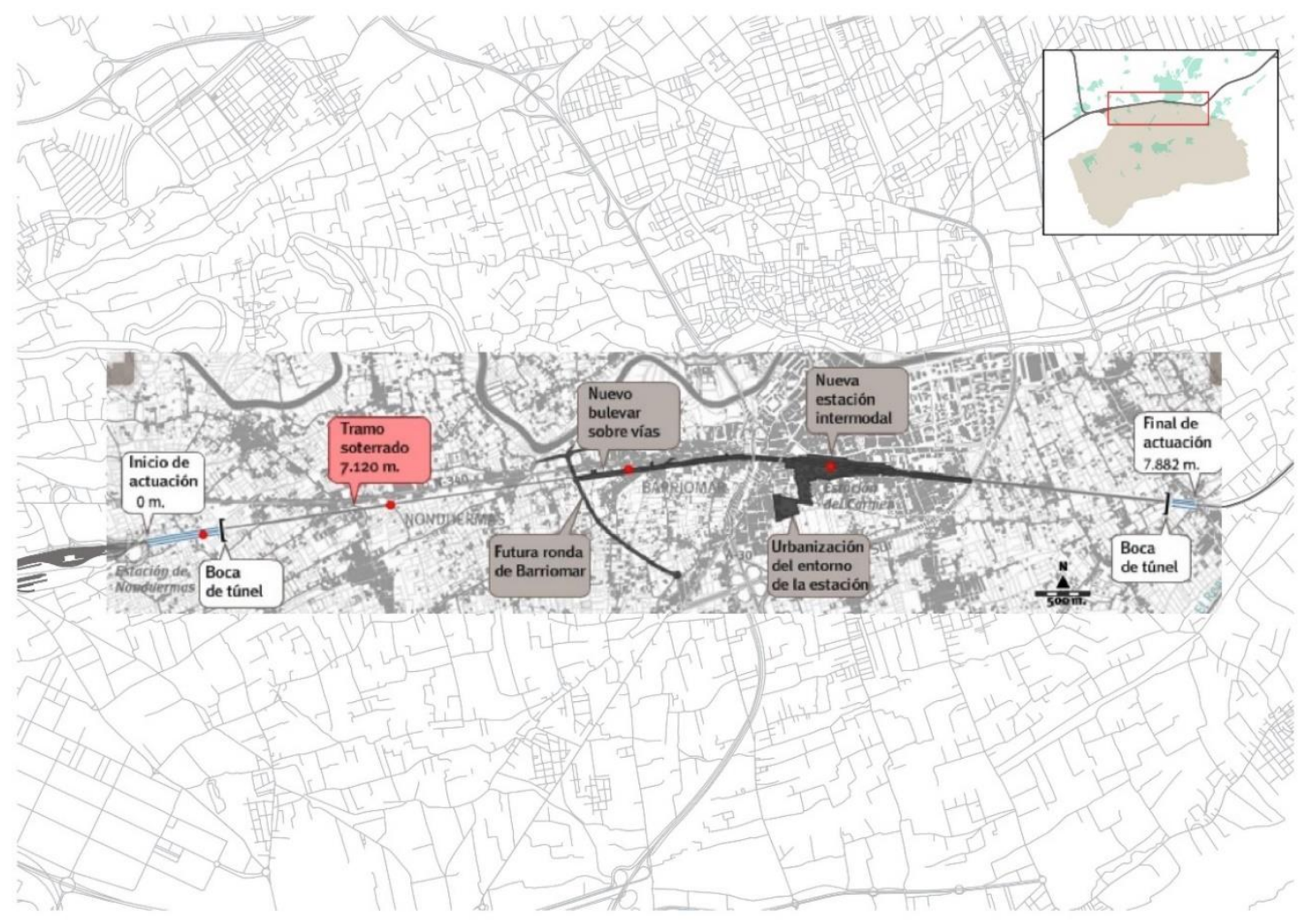

Fuente: Proyecto de Murcia Alta velocidad S.A. y elaboración propia

Este escenario de integración soterrada permite circular en superficie, tanto a vehículos como a peatones, sin la presencia de barreras de coste agregado o de restricción que se daban en los pasos a nivel que conllevaba el tránsito ferroviario en superficie.

\subsection{CÁlCULO DE VARIACIÓN DE COSTES}

El grado de vulnerabilidad se evalúa cuantificando los incrementos o disminuciones en los costes de desplazamiento, en términos de tiempo.

Mediante el empleo de ArcGIS 10.3. se realiza el cálculo de rutas de mínimo coste (en tiempo), obteniendo la matriz de coste de desplazamiento entre orígenes y destinos, a través de la funcionalidad OD Matrix Cost.

El análisis estadístico parte del cálculo porcentual de los modelos de inserción en superficie y soterrada respecto al modelo de referencia. De este modo se determina la variación porcentual (VP) del coste entre orígenes y destinos (coste OD) para el escenario en superficie respecto al de referencia, al igual que la variación porcentual para el coste OD correspondiente al escenario soterrado respecto al de referencia.

Por medio del procedimiento de interpolación IDW de ArcGIS 10.3. se elabora la cartografía. 


\section{RESULTADOS Y DISCUSIÓN}

Las representaciones cartográficas que se muestran a continuación, exponen la vulnerabilidad de la red a través de la polarización del área estudiada, como resultado de las variaciones en los costes de desplazamiento que se deducen del análisis comparativo de los escenarios de inserción de la AVF (en superficie y soterrada) con el escenario de referencia, previo a la inserción de la AVF.

La inserción en superficie supone para los desplazamientos a pie incrementos del 3,4\% en las zonas más afectadas.

FIGURA 5

Cartografía de variación porcentual. Escenario de inserción en superficie, movilidad a pie

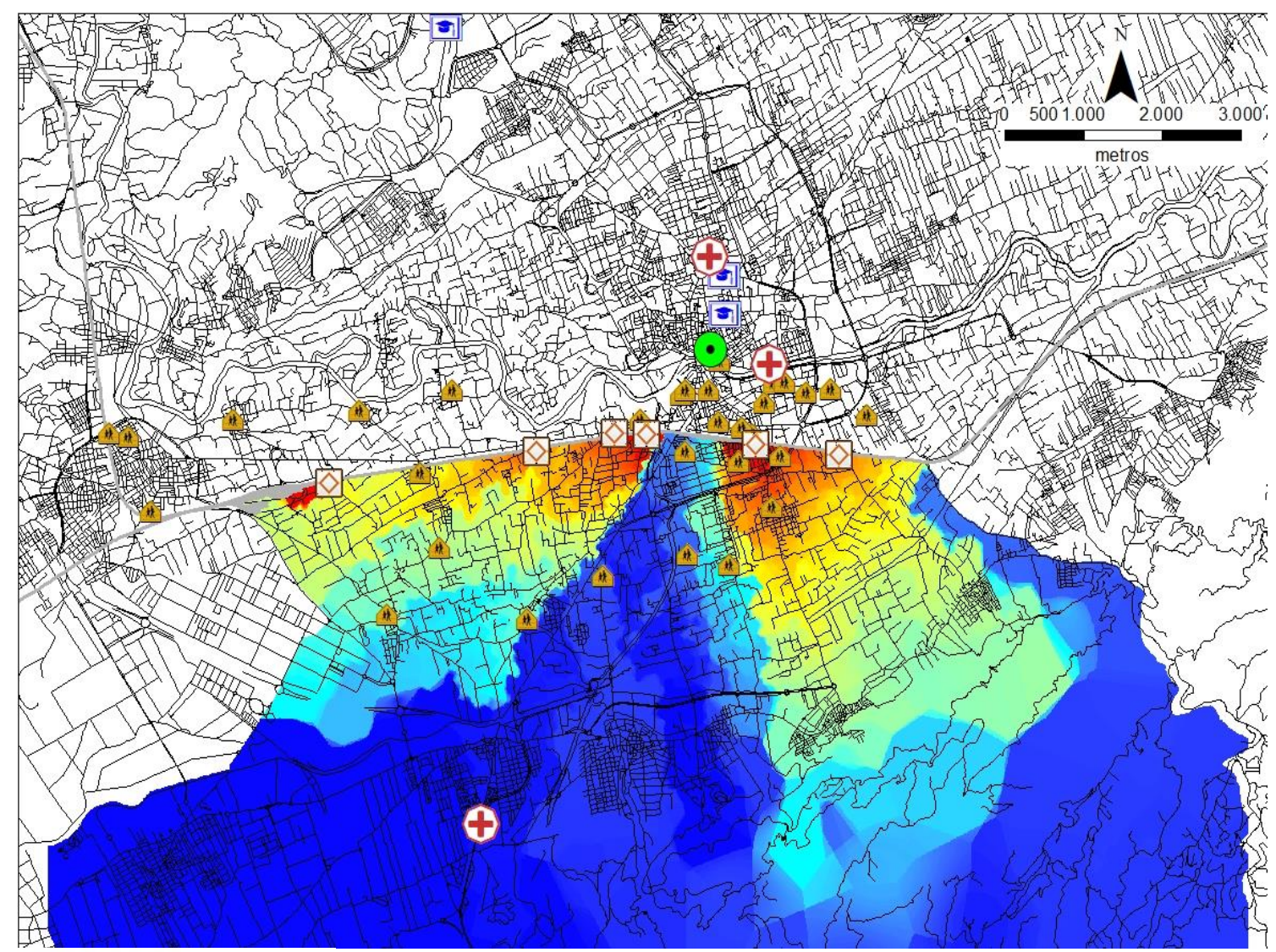

\section{LEYENDA}

$\%$

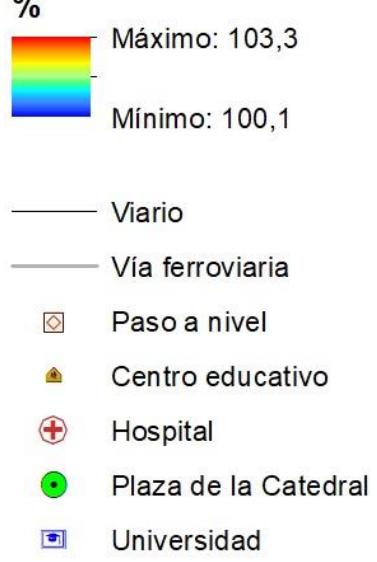

Fuente: Elaboración propia 
Según se observa en la figura 5, las áreas más vulnerables son las cercanas a pasos a nivel, hecho deducido por el incremento de coste que supone el tránsito por las pasarelas elevadas en lugar de por los pasos a nivel, influyendo, además, la cercanía a los destinos ya que el incremento respecto al coste total, se vuelve más notable, que en las áreas más alejadas de los destinos donde el incremento tiene escaso peso en el coste de desplazamiento.

Destaca la mínima variación que presenta la zona centro del área de estudio, este hecho se debe a que gran parte de los desplazamientos empleen la carretera del Palmar y atraviesen las vías por el paso inferior de calle Floridablanca, no viéndose afectado por los cambios generados de la inserción de la AVF en la ciudad.

La inserción en superficie presenta unos efectos muy diferentes en los desplazamientos mediante vehículos a motor, dada la circunstancia de que la conexión por medio de pasos a nivel quedaría inhabilitada, careciendo de alternativa al contrario de como sucede en el caso de los peatones con la existencia de pasarelas elevadas ubicadas donde los anteriores pasos a nivel como alternativa a ellos. Por tanto, los vehículos deben trazar sus rutas a través de los pasos inferiores o elevados que atraviesan las vías por debajo o por arriba de ellas, como son la autovía A-30, Ronda Sur y Camino de Tiñosa como pasos elevados, y carretera del Palmar, Camino Hondo y Avenida de Era Alta como pasos inferiores. La modificación de los itinerarios conlleva las variaciones en el coste de desplazamiento representadas a continuación:

FIGURA 6

Cartografía de variación porcentual. Escenario de inserción en superficie, movilidad mediante vehículo

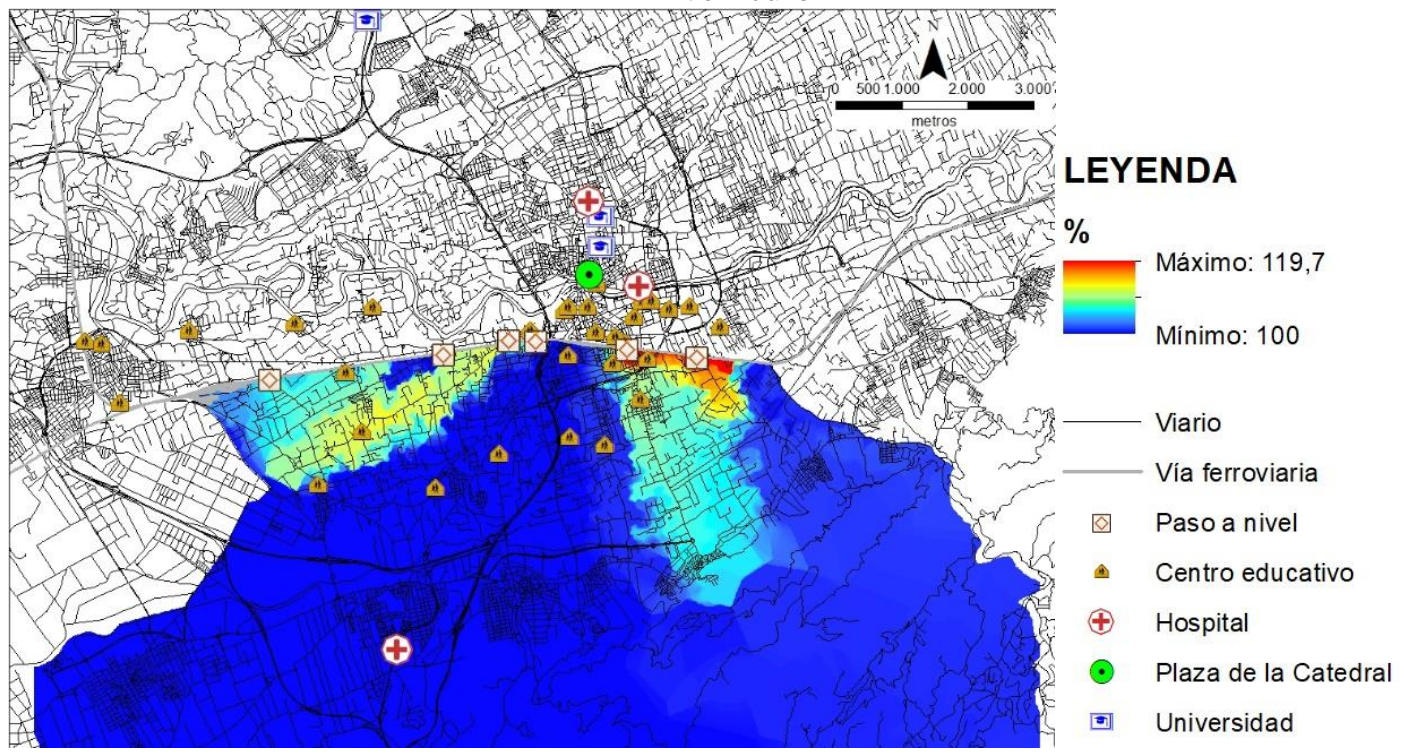

Fuente: Elaboración propia

La cartografía señala las áreas de la red más vulnerables, afectadas por un incremento considerable, distinguiéndose una franja con origen en el paso a nivel de Camino Baden, que se extiende en dirección SO hasta el núcleo urbano de San Ginés, abarcando el sur de la Era Alta. La variación en el coste de desplazamiento que sufriría la descrita banda sería consecuencia de la supresión del paso a nivel de Camino Baden.

Se observa incremento también en otras zonas próximas a pasos a nivel como es el caso del espacio adyacente a los pasos a nivel de Camino de Barreras y Barriomar, este incremento viene justificado por la dependencia hacia estos pasos en los trayectos a los destinos. No obstante, el espacio que presenta una mayor vulnerabilidad es el que se corresponde con el barrio de Santiago el Mayor junto a la zona adyacente al paso a nivel de Senda de Los Garres, el incremento se extiende desde estos pasos hasta la pedanía de Algezares, sin embargo, el incremento de coste disminuye con la distancia respecto a estos pasos. 
La cartografía también revela que ciertos espacios del área de estudio no presentan vulnerabilidad. Esto se explica debido a que los trayectos desde estas zonas hacia los destinos se realizan en ambos escenarios (escenario de inserción en superficie y escenario de referencia) a través de los pasos inferiores o elevados, resultando ajenos estos trayectos a la alteración que suscita la inserción de la AVF en superficie.

Una vez analizada la vulnerabilidad de la red y el territorio ante la inserción de la AVF en superficie, se da paso a exponer del mismo modo como afectaría la inserción, pero en este caso, soterrando las vías.

El estudio de la influencia sobre el sistema urbano que ejercería la integración de la AVF soterrada ha permitido conocer que dicha implantación supondría leves descensos en el coste de desplazamiento desde los espacios del área de estudio hacia los destinos. Permitiendo distinguir entre las zonas que muestran un mayor o menor descenso en el coste.

FIGURA 7

Cartografía de variación porcentual. Escenario de inserción soterrada, movilidad a pie

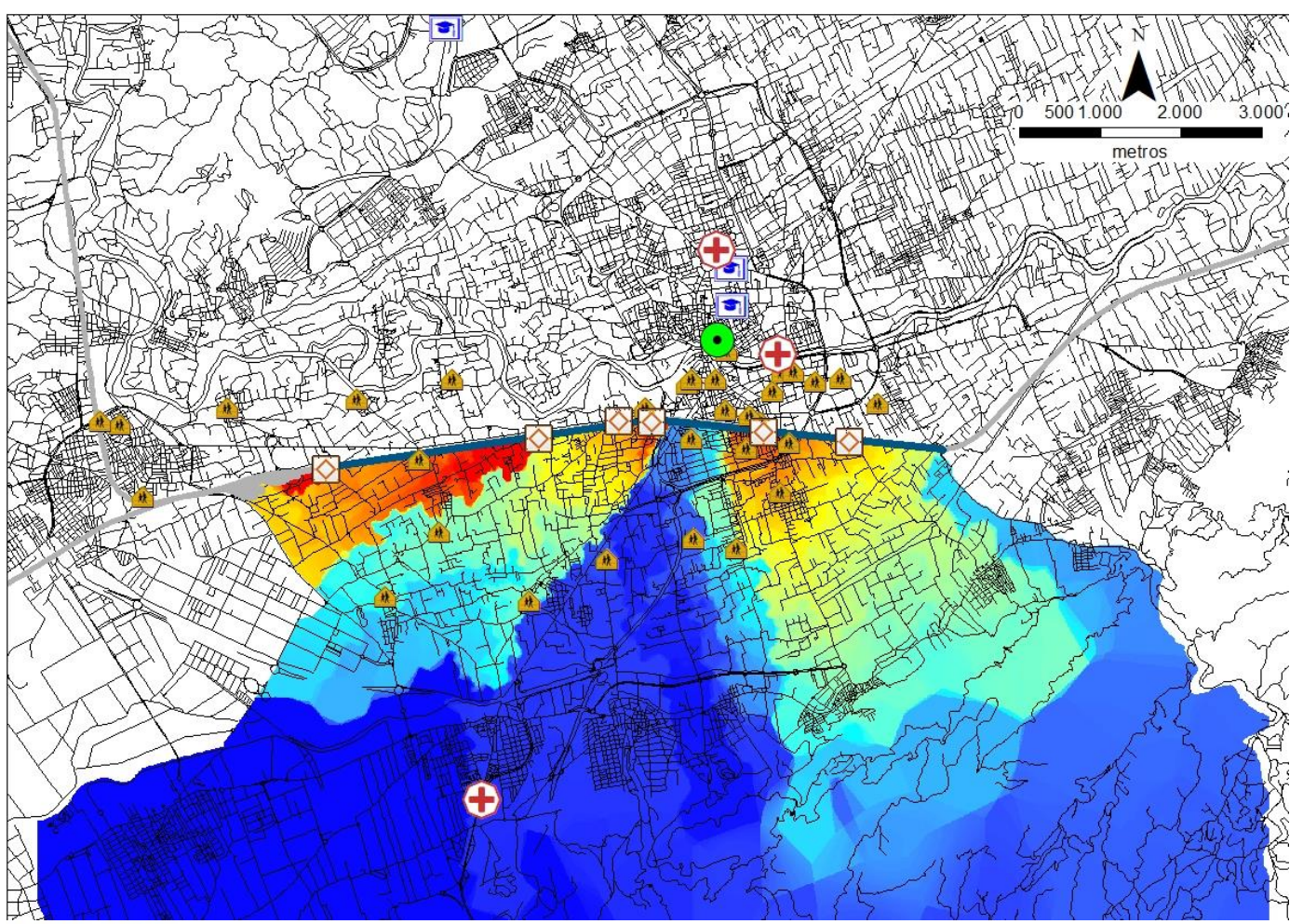

\section{LEYENDA}

$\%$

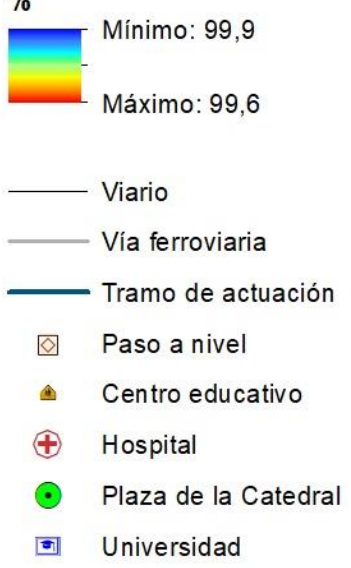

Fuente: Elaboración propia 
Esta disminución del coste de desplazamiento viene justificada por la eliminación de los tiempos de espera en los pasos a nivel debidos al tránsito ferroviario, que obligaba tanto a peatones como a vehículos a detenerse y esperar frente a las vías, para poder cruzarlas una vez el paso de ferrocarriles había finalizado. La inserción de la AVF soterrada significaría el tránsito de los trenes bajo tierra permitiendo la autónoma circulación de peatones y vehículos en superficie.

Los resultados revelan que esta nueva situación reduciría los tiempos de desplazamiento a pie, principalmente en zonas próximas a antiguos pasos a nivel, de entre las que destacan el Carril de Miajas y Camino de Barreras, además del sector contiguo al paso de Barriomar y el espacio próximo al paso de Santiago el Mayor.

Cabe señalar la escasa variación de coste que presenta el espacio central del área de estudio, correspondido a la carretera del Palmar, esta escasa variación se debe a la independencia de estas zonas con respecto a los pasos a nivel, dado que los desplazamientos hacia los destinos se realizan principalmente, por medio del paso inferior de carretera del Palmar con calle Floridablanca.

Por su parte, si se observa los efectos de la inserción soterrada para los desplazamientos mediante vehículo a motor, las variaciones de coste están, como ya se ha visto en los anteriores resultados, relacionadas con la proximidad o cercanía a los pasos a nivel.

FIGURA 8

Cartografía de variación porcentual. Escenario de inserción soterrada, movilidad en vehículo

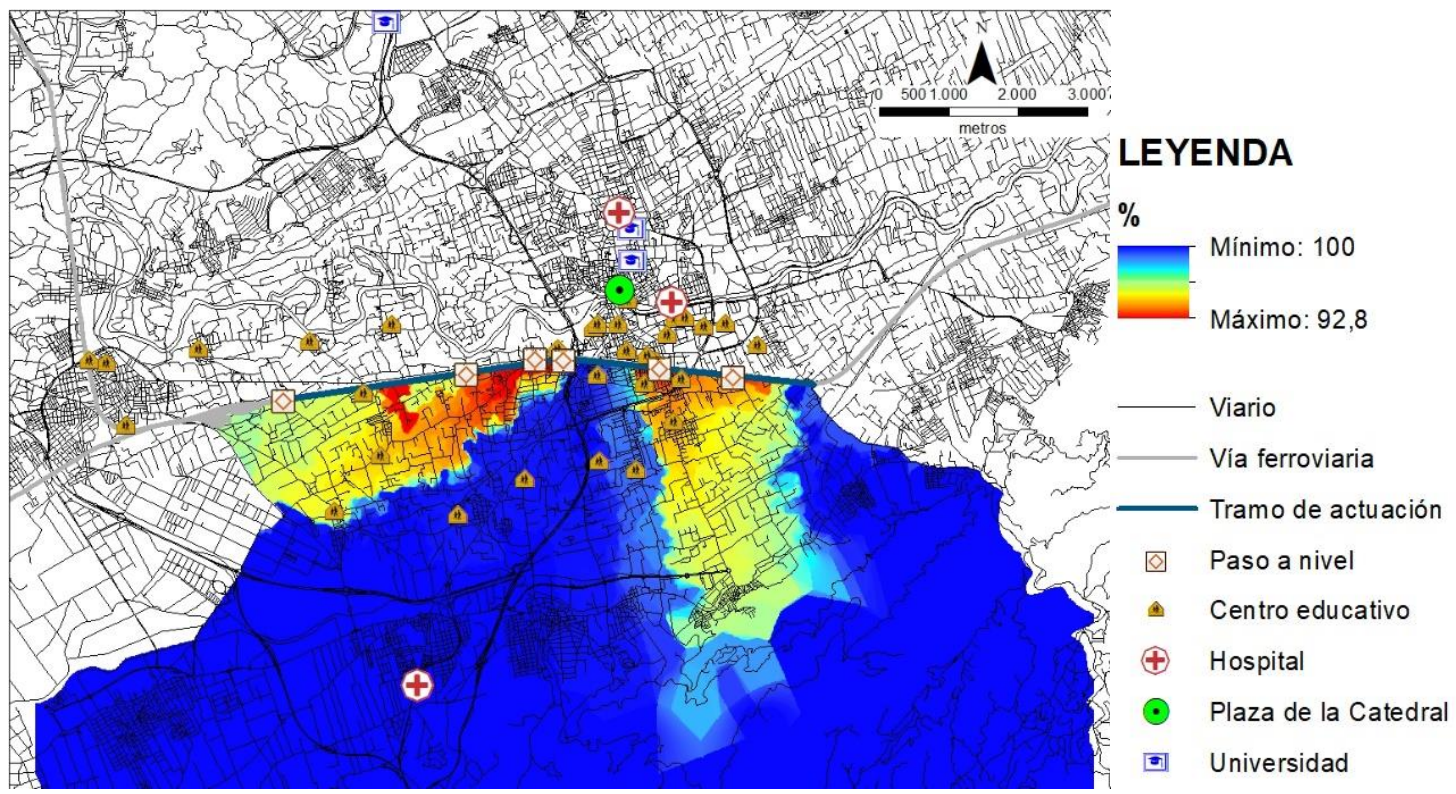

Fuente: Elaboración propia

Se distinguen zonas en las cuales la reducción del coste es considerablemente significativa, así sucede en el barrio de Santiago el Mayor dada su absoluta proximidad al paso a nivel conocido por el mismo nombre, además del espacio próximo al paso de Senda de los Garres, así como también se ve concernida con todavía mayor intensidad que las anteriores zonas, una franja que se extiende desde los pasos de Barriomar, Camino Albadel o Baden y Carril de Miajas en dirección suroeste hasta prácticamente alcanzar la pedanía de San Ginés, y que engloba todo el área de influencia de la carretera RM-611.

Una vez realizado el análisis espacial de la red de transporte identificando y diferenciando las zonas del área de estudio en función de su vulnerabilidad ante la inserción de la AVF, se da paso al análisis mediante cifras con el objetivo de cuantificar la población afectada por la alteración de la red.

Los resultados obtenidos revelan que la implantación de la AVF en superficie en contraste con la situación anterior (escenario de referencia) incrementa el coste para el 100\% de la población 
del territorio estudiado cuando ésta se desplaza a pie, sin embargo, la población afectada es de aproximadamente el $87 \%$ si los desplazamientos se realizan mediante vehículo a motor. En valores absolutos supone 113.065 personas afectadas para el caso de desplazamiento a pie y 97.760 personas para el caso de desplazamiento mediante vehículo a motor.

Las cifras de población afectada van variando en función del grado de incremento de coste, pues para el caso de los desplazamientos a pie un incremento del coste igual o superior al $1 \%$ afectaría al 48,6\% de la población del área de estudio (54.975 personas), mientras que, para el transporte en vehículo a motor, un incremento igual o superior al $4 \%$ supondría el $27,2 \%$ de la población del área de estudio afectada.

La población afectada por un incremento igual o superior al $50 \%$ del valor máximo de incremento es del 32,1\% (36.240 personas) para la modalidad de desplazamiento a pie y del $8,8 \%$ (9.950 personas) para la modalidad de desplazamiento en vehículo a motor.

Los valores de incremento más altos, iguales o superiores al 3\% para el caso de los peatones, e iguales o superiores al $19 \%$ para la modalidad de desplazamiento en vehículo a motor, los sufre el $12,5 \%$ de los peatones que se corresponde con 14.130 personas, y el 2,7\% de los conductores que se corresponde con 3.080 personas.

El estudio ha arrojado una serie de datos sobre cómo afecta a los desplazamientos la implantación de la AVF bajo soterramiento con respecto a la situación anterior a la llegada de la AVF, los datos muestran que esta integración disminuye los costes de desplazamiento a pie para el $100 \%$ de la población del área de estudio, mientras que la disminución del coste afecta al aproximadamente $87 \%$ de la población cuando ésta se desplaza mediante vehículo a motor.

Analizando la población afectada en función del grado de disminución de coste, se puede destacar que la reducción superior al 0'1\% del tiempo previo a la integración de la AVF soterrada afecta al $31 \%$ de la población (35.250 personas) cuando el desplazamiento es a pie; sin embargo, el decremento superior al 3\% del tiempo previo a la integración de la AVF soterrada incurre sobre el 33\% de la población del área de estudio (36.945 personas) en caso de desplazamiento mediante vehículo a motor.

Por otro lado, el decremento promedio, estimado en una disminución del 0'18\% del coste anterior a la integración de la AVF soterrada, afecta al 10\% de los peatones del área de estudio, que se corresponde con 11.610 habitantes; el decremento promedio para los desplazamientos en vehículo se estima en un 3,6\% y afecta al 27\% de la población del área de estudio, unas 30.520 personas.

Finalmente, el decremento más alto supera levemente el 0,3\% de tiempo de adelanto respecto al coste previo a la llegada de la AVF bajo soterramiento de las vías, afectando para el caso de la movilidad peatonal a un $1 \%$ de la población (1.400 personas); mientras que, en lo referido a la movilidad mediante vehículo, el decremento más destacado trata de un $7 \%$ de tiempo de adelanto respecto al coste previo a la llegada de la $\mathrm{AVF}$ incidiendo en un 3\% de la población del área de estudio, que se corresponde con 3.215 personas.

\section{CONCLUSIONES}

Se ha propuesto una metodología para desarrollar el análisis de la red de transporte del sur del municipio de Murcia. Basada en la formulación de escenarios (hipotéticos y reales) de evaluación y el cálculo de las variaciones en el tiempo de desplazamiento, mediante análisis de redes en un Sistema de Información Geográfica (ArcGIS).

Las diferencias observadas (pérdidas o ganancias de accesibilidad) entre cada uno de los escenarios de evaluación y el escenario de referencia (que refleja el funcionamiento de la red previo a la inserción de la AVF) permiten analizar la vulnerabilidad de la red y considerar los efectos sobre el territorio estudiado. La interpolación y el cálculo de variación porcentual del coste de desplazamiento permite conocer la intensidad de los efectos provocados por la inserción de la AVF en función de los escenarios. A partir de los resultados cartográficos y de las secciones censales se cuantifica la población afectada.

La metodología ha sido empleada para analizar la red de carreteras del sur del municipio de Murcia, teniendo en cuenta las características de los tramos. Las zonas más próximas a tramos sujetos a barreas de restricción o de coste añadido (pasos a nivel) son las que sienten mayores 
variaciones de accesibilidad, conforme al alejamiento de estos tramos la variación se va reduciendo.

Los resultados permiten afirmar que la inserción de la Alta Velocidad Ferroviaria en el tejido de esta red de transporte implica variaciones destacadas en los tiempos de desplazamiento. Las variaciones resultan positivas en el caso de una integración soterrada pues disminuye los tiempos de desplazamiento y mejora la accesibilidad de la red. Mientras que una inserción en superficie significaría incrementos en los tiempos resultando negativa para la funcionalidad de la red.

Se afirma pues, que la integración en superficie produce alteraciones negativas en la red, debido a los retrasos que produciría tanto en el tráfico de vehículos como en el peatonal. Existiendo verosimilitud en que produzca un efecto de degradación de la calidad de vida y pérdida de valor del suelo en las zonas identificadas, en este trabajo, como más vulnerables.

La conexión de la ciudad de Murcia con el resto de áreas del país por medio de la AVF, puede suponer un impulso para la ciudad y para toda la región murciana en lo referente al desarrollo económico, principalmente para los sectores secundarios y terciarios, dado que esta mejora de la comunicación facilita los desplazamientos con fines empresariales o turísticos, entre otros.

La mejora de la comunicación ferroviaria con el resto del país no es la única o exclusiva ventaja que puede conllevar la llegada de la AVF a la capital murciana, pues la integración puede, como se ha demostrado en este trabajo, mejorar la propia comunicación, articulación y accesibilidad de la red, si esta inserción se realiza soterrando las vías. Los análisis de vulnerabilidad de las redes de transporte, tal y como se ha observado en este ejemplo de metodología y aplicación, aportan información de interés, en los proyectos de gestión y planificación del transporte y ordenación del territorio.

\section{BIBLIOGRAFÍA}

ALBERT, R., HAWOONG, J. y BARABASI, A. L. (2000): "Error and attack tolerance of complex networks". Nature 406: 378-382.

ÁLVAREZ, E. (2016): "Ferrocarril y sistema de ciudades. Integración e impacto de las redes ferroviarias en el contexto urbano europeo". Biblio 3W, 21(1.169).

ÁLVAREZ, E. y HERNÁNDEZ, M. (2012): "La infraestructura ferroviaria como condicionante del crecimiento de la trama urbana en ciudades medianas catalanas a principios del siglo XX". In VI Congreso de Historia Ferroviaria, Vitoria-Gasteiz, 2012 (pp. 1-22). Fundación de los Ferrocarriles Españoles.

BALL, M. O., GOLDEN, B. L. y VOHRA, R. V. (1989): "Finding the most vital arcs in a network". Operations Research Letters 8, 73-76.

BEL, C. y GÓMEZ, J. (1998): "Indicadores demográficos y su distribución territorial en el municipio de Murcia". Papeles de Geografía, (28).

BELLET, C., ALONSO, P. y CASELLAS, A. (2010): "Infraestructuras de transporte y territorio. Los efectos estructurantes de la llegada del tren de alta velocidad en España". Boletín de la Asociación de Geógrafos Españoles, 2010, núm. 52, p. 143-163.

BELLET, C. y GUTIÉRREZ, A. (2011): "Ciudad y ferrocarril en la España del siglo XXI. La integración de alta velocidad ferroviaria en el medio urbano". Boletín de la Asociación de Geógrafos Españoles, 2011, núm. 55, p. 251-279.

BERDICA, K. y MATTSSON, L.G. (2007): "Vulnerability: A Model-Based Case Study of the Road Network in Stockholm, en Murray. A. and Grubesic, T. (Eds.): Critical Infrastructure. Reliability and Vulnerability". Berlin, Springer, 81-106.

BONO, F. y GUTIÉRREZ, E. (2011): "A network-based analysis of the impact of structural damage on urban accessibility following a disaster: the case of the seismically damaged Port Au Prince and Carrefour urban road networks". Journal of Transport Geography, 19, 14431455.

COVA, T. J. (1999): "GIS in emergency management". Geographical Information Systems, 2, 845-858.

DERRIBLE, S. y KENNEDY, C. (2011): "Applications of Graph Theory and Network Science to Transit Network Design". Transport Reviews: A Transnational Transdisciplinary Journal, 31(4), pp. 495-519. 
ERATH, A., BIRDSALL, J., AXHAUSEN, K.W. y HAJDIN R. (2008): "Vulnerability assessment of the Swiss road network". Research Report. Eidgenössische Technische Hochschule, Institut für Verkehrsplanung und Transportsysteme (ETH-IVT), Zurich.

GARCÍA, F. M. (2012): Dinámicas de crecimiento de la Huerta de Murcia y similitudes con el Véneto italiano.

GARRISON, W. L. (1960): “Connectivity of the Interstate Highway System”. Papers in Regional Science 6, 121-137.

GRADILLA-HERNÁNDEZ, L. A., LA LLATA-GÓMEZ, D. y GONZÁLEZ-GÓMEZ, o. (2011): "Índices de vulnerabilidad de redes carreteras. Enfoques recientes y propuesta de aplicación en México". Ingeniería, investigación y tecnología, 12(3), 257-267.

GUTIÉRREZ, J. (2001): "Location, economic potential and daily accessibility: an analysis of the accessibility impact of the high-speed line Madrid-Barcelona-french border". Journal of Transport Geography, 9, 229-242.

JENELIUS, E. y MATTSSON, L.G. (2005): "Developing a methodology for road network vulnerability análisis". Nectar Cluster 1 Seminar, 12th - 13th May 2006, Molde University College, Molde (Norway).

KNOOP, V., ZUYLEN, H. y HOOGENDOORN, S. (2008): "The influence of spillback modelling when assessing consequences of blockings in a road network". European Journal of Transportation and Infrastructure Research 8, 287-300.

MURRAY, A. T., MATISZIW, T. C. y GRUBESIC, T. H. (2008): “A methodological overview of network vulnerability análisis". Growth and Change 39, 573-592.

NAGURNEY, A. y QIANG, Q. (2011): "Fragile Networks: Identifying Vulnerabilities and Synergies in an Uncertain Age". International Transactions in Operational Research, 19(1-2), pp. 123-160.

NEWMAN, M. (2010): "Networks: an introduction". New York, New York, USA: Oxford University Press. 784p

GOL'DSHTEIN, V., KOGANOV, G. A. y SURDUTOVICH, G. I. (2004): Vulnerability and hierarchy of complex networks.

PÉREZ, F. (1994): “La integración del ferrocarril en la ciudad”. Revista de Obras Públicas, 3, 7989.

RATLIFF, H. D., SICILIA, G. T. y LUBORE, S. H. (1975): "Finding the n most vital links in flow networks". Management Science 21, 531-539.

RESTREPO, J. H. y SÁNCHEZ, J. J. (2004): “Aplicación de la teoría de grafos y el algoritmo de Dijkstra para determinar las distancias y las rutas más cortas en una ciudad". Scientia et technica, 10(26).

RODRÍGUEZ, E. y GUTIÉRREZ, J. (2012): “Análisis de vulnerabilidad de redes de carreteras mediante indicadores de accesibilidad y SIG: Intensidad y polarización de los efectos del cierre de tramos en la red de carreteras de Mallorca". GeoFocus. Revista Internacional de Ciencia y Tecnología de la Información Geográfica, (12), 374-394. ISSN: 1578-5157.

ROS, M., SANZ, J. P. y GARCÍA, F. M. (2010): La gestión del territorio periurbano en la huerta de Murcia.

SANTOS, L. (2007): Urbanismo y ferrocarril. La construcción del espacio ferroviario en las ciudades medias españolas. Madrid: Fundación de los Ferrocarriles Españoles.

SANTOS, L. y DE LAS RIVAS, J. L. (2005): El proyecto urbanístico del AVE en Valladolid. Ingeniería y territorio, (70), 88-95.

TAYLOR, M. A. P., SEKHAR, S. V. C. y D’ESTE, G. M. (2006): “Application of Accessibility Based Methods for Vulnerability Analysis of Strategic Road Networks". Networks \& Spatial Economics, 6, 267-291.

VÉLEZ, L. L. y HEREDIA, R. G. H. (2014): "Vulnerabilidad de redes complejas y aplicaciones al transporte urbano: una revisión de la literatura". Revista EIA, 11(21), 67-77. 